\title{
ASSOCIAÇÃO ENTRE SINTOMAS RESPIRATÓRIOS, DADOS SOCIODEMOGRÁFICOS E ANTROPOMETRIA EM AGRICULTORES FAMILIARES
}

\section{ASSOCIATION BETWEEN RESPIRATORY SYMPTOMS, SOCIODEMOGRAPHIC DATA AND ANTHROPOMETRY IN FAMILY FARMERS}

\begin{abstract}
lanka Quirino Soares dos Santos ${ }^{1}$, Marta Ligia Vieira Melo², Kamilla Zenóbya Ferreira Nóbrega de Souza ${ }^{3}$,
\end{abstract} Elisangela Vilar de Assis ${ }^{4}$, Ubiraídys de Andrade Isidório ${ }^{5}$

Submetido: 08/12/2020

Aprovado: 22/01/2021

\begin{abstract}
RESUMO
INTRODUÇÃO: Cada país tem sua economia e grande parte dela advém do desenvolvimento do trabalho agrícola, responsável por atender às necessidades da população. Essa prática contribui de forma positiva, pois ajuda na renda familiar, mas também apresenta uma vertente negativa aos agricultores, pois a maioria trabalha de forma inadequada para sua segurança, correndo o risco de adquirir doenças respiratórias ocupacionais. Objetivo: Esta pesquisa teve como objetivo analisar a associação entre sintomas respiratórios, dados sociodemográficos e antropometria em agricultores. Materiais e Métodos: Essa abordagem ocorreu na residência dos entrevistados, durante os meses de fevereiro e março de 2020. Participaram da pesquisa agricultores de ambos os sexos, a partir de 18 anos de idade. Foram excluídos os que tiveram menos de seis meses de trabalho na agricultura e os que possuíram doença respiratória diagnosticada antes do início do trabalho na agricultura. Foram coletados dados referentes às informações sociodemográficas, sintomas respiratórios e dados antropométricos. Resultados: Observou-se que, entre os mais velhos, a maioria é analfabeta, trabalha há mais de 20 anos e possui carga horária semanal de mais de 60 horas. Os jovens e os idosos têm as medidas antropométricas diferentes comparadas com a idade, não sendo constatada correlação entre dados antropométricos e sintomas respiratórios nas pessoas mais jovens. Por fim, mais tempo de trabalho esteve relacionado a sibilos e tosse por pelo menos três meses. Conclusão: Concluiu-se que é necessária a assistência para a saúde desses trabalhadores, com estratégias de promoção e prevenção a saúde para prevenção das doenças respiratórias.
\end{abstract}

PALAVRAS-CHAVE: Agricultura. Saúde do trabalhador. Sintomas respiratórios

\begin{abstract}
Introduction: Each country has its economy and a large part of it comes from the development of agricultural work, responsible for meeting the needs of the population. This practice contributes in a positive way, as it helps in the family income, but it also presents a negative aspect to the farmers, since the majority works in an inadequate way for their safety, running the risk of acquiring occupational respiratory diseases. Objective: To analyze the association between respiratory symptoms, sociodemographic data, and anthropometry in family farmers. Materials and Method: The

\footnotetext{
1 Fisioterapeuta graduada pela Faculdade Santa Maria - FSM, Cajazeiras, Paraíba. E-mail: iankaquirino@gmail.com

2 Mestre em Saúde Coletiva pela Universidade de Santos (UNISANTOS), Santos, São Paulo. Professora da Faculdade Santa Maria (FSM), Cajazeiras, Paraíba, Brasil. E-mail: martaligiafisio@hotmail.com

3 Especialista em Fisioterapia em Terapia Intensiva pela Sociedade Brasileira de Terapia Intensiva (SOBRATI); Professora da Faculdade Santa Maria (FSM), Cajazeiras, Paraíba, Brasil. E-mail: kamillazenobya@hotmail.com

4 Doutora em Ciências da Saúde pela Faculdade de Medicina do ABC, Santo André, São Paulo. Professora adjunta da Universidade Federal de Campina Grande - UFCG, Campus Cajazeiras, Paraíba. E-mail: ely.vilar@hotmail.com

5 Doutorando em Ciências da Saúde pela Faculdade de Medicina do ABC, Santo André, São Paulo. Professor da Faculdade Santa Maria - FSM, Cajazeiras, Paraíba. E-mail: ubiraidys_1@hotmail.com
} 


\title{
RECIMA21 - REVISTA CIENTÍFICA MULTIDISCIPLINAR
}

\author{
ASSOCIAÇÃO ENTRE SINTOMAS RESPIRATÓRIOS, DADOS SOCIODEMOGRÁFICOS E \\ ANTROPOMETRIA EM AGRICULTORES FAMILIARES \\ lanka Quirino Soares dos Santos, Marta Ligia Vieira Melo, Kamilla Zenóbya \\ Ferreira Nóbrega de Souza, Elisangela Vilar de Assis, Ubiraídys de Andrade Isidório
}

research was carried out at the farmers' homes. This approach occurred during the months of February and March 2020. Participants in the survey were farmers of both sexes, from 18 years of age. Those who had less than six months of work in agriculture, those who had respiratory disease diagnosed before starting work in agriculture were excluded. Data were collected regarding sociodemographic information, respiratory symptoms, and anthropometric data. Results: It was observed that, among the elderly, the majority is illiterate, has worked for more than 20 years, and has a weekly workload of more than 60 hours. Young people and the elderly have different anthropometric measures compared to age, with no correlation between anthropometric data and respiratory symptoms in younger people. Finally, longer working hours were related to wheezing and coughing for at least three months. Conclusion: Health care for these workers is necessary, with health promotion and prevention strategies to prevent respiratory diseases.

KEYWORDS: Agriculture. Worker's health. Respiratory symptoms.

\section{INTRODUÇÃO}

Cada país tem sua economia, e grande parte dela decorre do desenvolvimento de trabalhos agrícolas, que ajuda nas necessidades da população. Essa prática contribui de forma positiva, pois ajuda na renda familiar, mas também influi de forma negativa aos agricultores (BORGES et al., 2016).

A saúde da população é determinada pelos seus hábitos de vida, os que moram no ambiente rural têm baixo nível de escolaridade e, por isso, muitos atuam laboralmente de forma inadequada para sua segurança, sem ter consciência dos malefícios que podem ser causados (LIMA et al.,2015).

A agricultura está crescendo cada vez mais e, com isso, o número de agricultores aumenta, mas infelizmente crescem junto as doenças respiratórias que, com o passar dos anos, só piora a saúde dos trabalhadores (FONTOURA, ZANAGA, 2014). No Brasil são mais de 30 milhões de pessoas que vivem em condições de risco, fato que contribui para piores situações de insegurança durante a jornada de trabalho (LIMA et al.,2015).

As doenças respiratórias ocupacionais são causadas geralmente pela poluição do ar, o qual o trabalhador está exposto durante as diversas horas que permanece trabalhando. Tem-se que a exposição maléfica depende de vários fatores, podendo-se destacar os efeitos dos poluentes e o tabagismo (RONDON; GONÇALVES; BOTELHO, 2011).

Em todo o mundo a diversidade de substâncias químicas produzidas vem aumentando, gerando uma grande exposição do homem, tanto pela frequência de uso, como pela diversidade de substâncias existentes (FONTOURA et al., 2014).

Como exemplo, tem-se que o Brasil é o maior exportador mundial da folha de tabaco, esse é um dos trabalhos mais prejudiciais para a saúde dos trabalhadores e da população que, por efeito do contato com a poeira da folha do tabaco, torna-se vulnerável a intoxicações por agrotóxicos (LISBOA; AZEVEDO, 2014).

Os agrotóxicos são usados com frequência na agricultura. Os agricultores, mesmo sendo 


\title{
RECIMA21 - REVISTA CIENTÍFICA MULTIDISCIPLINAR
}

\author{
ASSOCIAÇÃO ENTRE SINTOMAS RESPIRATÓRIOS, DADOS SOCIODEMOGRÁFICOS E \\ ANTROPOMETRIA EM AGRICULTORES FAMILIARES \\ lanka Quirino Soares dos Santos, Marta Ligia Vieira Melo, Kamilla Zenóbya \\ Ferreira Nóbrega de Souza, Elisangela Vilar de Assis, Ubiraídys de Andrade Isidório
}

informados sobre os efeitos negativos que eles causam à saúde, por dificuldade de compreensão, acabam por ignorar o assunto (GREGOLIS; PINTO; PERES, 2012).

Os trabalhadores que ficam mais expostos aos diversos fatores de agravos a saúde são, em sua maioria, do sexo masculino, e quando aparecem alguns sintomas respiratórios não procuram o serviço de saúde, pelo fato de serem vistos pela sociedade como referência de força e trabalho, assim, acabam não se cuidando e, em decorrência de tal fato ocorre o aumento do número de mortalidade (FERRAZ et al., 2018). O trabalho agrícola, em muitos casos, prejudica não só o trabalhador, como também os seus familiares: filhos, esposas e idosos, que acabam expondo-se e adquirindo problemas respiratórios (GREGOLIS; PINTO; PERES, 2012).

As ações de promoção, proteção, tratamento e recuperação da saúde devem ser analisadas segundo o contexto social local das demandas, pois trabalhadores e moradores da zona rural possuem características distintas na sua forma de viver, trabalhar e se relacionar com o ambiente (LIMA et al.,2015)

Portanto, os programas de saúde devem viabilizar estratégias preventivas, principalmente direcionadas ao público masculino, direcionando ações singulares em conformidade com a vida, o ambiente de trabalho e o sexo, para colher todas as informações de cada pessoa, e assim contribuir com a redução dos agravos e mortalidade (FERRAZ et al.,2018).

Assim, a pesquisa objetivou analisar a associação entre sintomas respiratórios, dados sociodemográficos e antropometria de agricultores familiares.

\section{MÉTODO}

A pesquisa em questão segue um delineamento quantitativo, possui desenho transversal e analítico, e seguiu o método Snowball.

De acordo com o censo agropecuário do IBGE tem-se, aproximadamente, 1.082 pessoas ocupadas na agricultura familiar de Tenente Ananias-RN (BRASIL, 2006b), vale salientar ainda que, segundo a Declaração de Aptidão ao Pronaf - DAP (BRASIL, 2019), o respectivo município possui cerca de 1069 (Mil e sessenta e nove) registros junto à Secretaria Especial de Agricultura Familiar e do Desenvolvimento Agrário - SEAD.

A pesquisa foi desenvolvida nas residências dos agricultores, especificamente na zona rural do município de Tenente Ananias-RN, pertencente ao estado do Rio Grande do Norte.

Os participantes foram selecionados de forma aleatória e por conveniência. Foram avaliados cerca de 100 agricultores, encontrados por meio da indicação de outros agricultores. Essa abordagem ocorreu durante os meses de fevereiro e março de 2020 , aos sábados e domingos, das $7 \mathrm{~h} 30$ às $11 \mathrm{~h}$, no turno da manhã, e no período da tarde das $14 \mathrm{~h} 30 \mathrm{~min}$ às $17 \mathrm{~h} 00 \mathrm{~min}$. 
Participaram da pesquisa agricultores de ambos os sexos, a partir de 18 anos de idade. $\mathrm{E}$ foram excluídos os que tinham menos de seis meses de trabalho na agricultura e os que possuíam doença respiratória diagnosticada antes do início do trabalho na agricultura.

Os pesquisadores aplicaram um questionário semiestruturado, desenvolvido a partir do estudo de Guimarães; Oliveira (2015); Hoepers (2015); Buralli (2016); Farias (2012), no qual constaram questionamentos básicos, integrando perguntas como: idade, escolaridade, alfabetização, estado civil, renda mensal.

Além disso, foi empregado outro questionário acerca dos sintomas respiratórios, conhecido por European Community Respiratory Health Survey - ECRHS, optando como referência o modelo aplicado por Freitas (2017), o qual se propõe a avaliar a prevalência de sintomas respiratórios, através de um inquérito comportando nove questões voltadas para as manifestações respiratórias dos últimos meses e/ou anos.

Antecedentemente a aplicação dos questionários foi feita a entrega do Termo de Consentimento Livre e Esclarecido - TCLE para cada entrevistado, após esclarecimentos, os que se mostravam cientes e de acordo com os termos da pesquisa foram convidados a participar da pesquisa formalmente por meio da assinatura do termo ou colocação da impressão digital.

Esse trabalho faz parte da pesquisa intitulada "Trabalho Rural: associação entre exposições, sintomas respiratórios e qualidade do sono em agricultores familiares", aprovado pelo Comitê de Ética em Pesquisa (CEP) da Faculdade Santa Maria sob o parecer ํㅡ 3.551.893, obedecendo cautelosamente às normas previstas na Resolução 466/12 do Conselho Nacional de Saúde (BRASIL, 2012).

Os dados foram analisados no SPSS (versão 25). Além de estatística descritiva de frequência relativa e absoluta, medida de tendência central e de dispersão, utilizou-se testes inferenciais de correlação de Pearson e point bisserial. A significância estatística aceita foi de $p \leq$ 0,05 .

\section{RESULTADOS}

A tabela 1 mostra que entre os mais jovens há maioria de pessoas com renda de menos de um salário mínimo e entre os mais velhos, mais de 02 salários mínimos. Entre os mais velhos, a maioria das residências tem entre 01 e 02 moradores, é constituída por analfabetos que trabalham há mais de 20 anos e laboram com carga horária semanal entre 40 e 50 horas.

Tabela 1 - Características sociodemográficas dos agricultores divididos por idade.

\begin{tabular}{ccc}
\hline Variáveis & $\begin{array}{c}\text { Entre } 32 \text { e } 59 \\
\text { anos }\end{array}$ & $\begin{array}{c}\text { Entre } 60 \text { e } 88 \\
\text { anos }\end{array}$ \\
\hline
\end{tabular}


ASSOCIAÇÃO ENTRE SINTOMAS RESPIRATÓRIOS, DADOS SOCIODEMOGRÁFICOS E ANTROPOMETRIA EM AGRICULTORES FAMILIARES

lanka Quirino Soares dos Santos, Marta Ligia Vieira Melo, Kamilla Zenóbya Ferreira Nóbrega de Souza, Elisangela Vilar de Assis, Ubiraídys de Andrade Isidório

\begin{tabular}{|c|c|c|c|c|}
\hline & $\mathbf{F}$ & $\%$ & $\mathbf{F}$ & $\%$ \\
\hline \multicolumn{5}{|c|}{ № de moradores por residência } \\
\hline Entre 01 e 02 & 17 & 32,00 & 12 & 40,00 \\
\hline Entre 03 e 04 & 30 & 56,60 & 18 & 48,90 \\
\hline Entre 04 e 07 & 6 & 11,40 & 5 & 11,10 \\
\hline \multicolumn{5}{|l|}{ Renda familiar bruta } \\
\hline <1 Salário mínimo & 40 & 75,5 & 10 & 22,2 \\
\hline 22 Salário mínimo & 10 & 18,9 & 28 & 62,2 \\
\hline 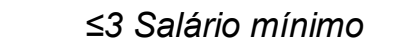 & 3 & 5,7 & 7 & 15,6 \\
\hline \multicolumn{5}{|l|}{ Escolaridade } \\
\hline Alfabetizado & 22 & 41,5 & 4 & 8,9 \\
\hline Não-alfabetizado & 31 & 58,5 & 41 & 91,1 \\
\hline \multicolumn{5}{|l|}{ Tempo de trabalho } \\
\hline Entre 15 e 20 anos & 4 & 7,5 & 0 & 0,0 \\
\hline$>20$ anos & 49 & 92,5 & 45 & 100,0 \\
\hline \multicolumn{5}{|c|}{ Carga horária semanal de trabalho } \\
\hline Entre 20 e 35 horas & 6 & 11,3 & 2 & 4,4 \\
\hline Entre 40 e 50 horas & 29 & 54,7 & 24 & 53,3 \\
\hline$>60$ horas & 18 & 34,0 & 19 & 42,2 \\
\hline
\end{tabular}

A tabela 2 mostra que os jovens e os idosos têm as medidas antropométricas diferentes comparadas com a idade, em que os maiores valores são: os jovens têm a circunferência abdominal de 97,96, altura 161,43 e circunferência de quadril 101,96, e os idosos possuem circunferência abdominal 101,89, altura 161,98 e circunferência de quadril 100,13.

Tabela 2 - Características das medidas antropométricas em relação a idade

\begin{tabular}{lcccc}
\hline \multirow{2}{*}{ Variáveis } & \multicolumn{2}{c}{ Entre 32 e 59 anos } & \multicolumn{2}{c}{ Entre 60 e 88 anos } \\
\cline { 2 - 5 } & Média & $\begin{array}{c}\text { Desvio } \\
\text { padrão }\end{array}$ & Média & $\begin{array}{c}\text { Desvio } \\
\text { padrão }\end{array}$ \\
\hline Peso & $71,88 \mathrm{~kg}$ & $13,37 \mathrm{~kg}$ & $71,72 \mathrm{~kg}$ & $16,42 \mathrm{k}$ \\
Circunferência Abdominal & $\mathbf{9 7 , 9 6 \mathrm { cm }}$ & $10,69 \mathrm{~cm}$ & $\mathbf{1 0 1 , 8 9 c m}$ & $14,61 \mathrm{~cm}$ \\
Relação Cintura/ Quadril & $90,92 \mathrm{~cm}$ & $6,52 \mathrm{~cm}$ & $94,42 \mathrm{~cm}$ & $7,35 \mathrm{~cm}$ \\
Altura & $\mathbf{1 6 1 , 4 3 m}$ & $8,85 \mathrm{~m}$ & $\mathbf{1 6 1 , 9 8 m}$ & $6,72 \mathrm{~m}$ \\
Circunferência da cintura & $92,77 \mathrm{~cm}$ & $9,49 \mathrm{~cm}$ & $94,64 \mathrm{~cm}$ & $9,84 \mathrm{~cm}$ \\
IMC & $27,46 \mathrm{Kg} / \mathrm{m}^{2}$ & $4,68 \mathrm{Kg} / \mathrm{m}^{2}$ & $27,29 \mathrm{Kg} / \mathrm{m}^{2}$ & $5,34 \mathrm{Kg} / \mathrm{m}^{2}$ \\
Circunferência do quadril & $\mathbf{1 0 1 , 9 6 \mathbf { c m }}$ & $\mathbf{8 , 0 6 \mathrm { cm }}$ & $\mathbf{1 0 0 , 1 3 \mathbf { c m }}$ & $9,10 \mathrm{~cm}$ \\
\hline
\end{tabular}

A tabela 3 não constatou correlação entre dados antropométricos e sintomas respiratórios nas pessoas mais jovens. Para as pessoas mais velhas, verificaram-se correlações estatisticamente significativas, principalmente nos sintomas de alergia no nariz e tosse por pelo menos 03 meses. Alergia no nariz e tosse por pelo menos 03 meses mostraram relação com maiores medidas antropométricas.

Tabela 3 - Correlações dos sintomas respiratórios com os dados antropométricos em função da idade 
ASSOCIAÇÃO ENTRE SINTOMAS RESPIRATÓRIOS, DADOS SOCIODEMOGRÁFICOS E ANTROPOMETRIA EM AGRICULTORES FAMILIARES lanka Quirino Soares dos Santos, Marta Ligia Vieira Melo, Kamilla Zenóbya Ferreira Nóbrega de Souza, Elisangela Vilar de Assis, Ubiraídys de Andrade Isidório

\begin{tabular}{|c|c|c|c|c|c|c|c|c|}
\hline $\begin{array}{l}\frac{0}{2} \\
\frac{\pi}{0} \\
\end{array}$ & & @ & 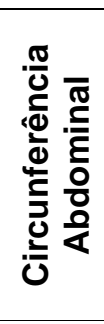 & 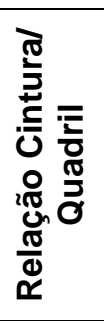 & $\stackrel{\frac{\pi}{5}}{\frac{\pi}{4}}$ & 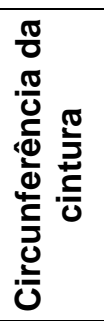 & $\underline{\underline{U}}$ & 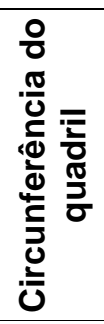 \\
\hline \multirow{11}{*}{ 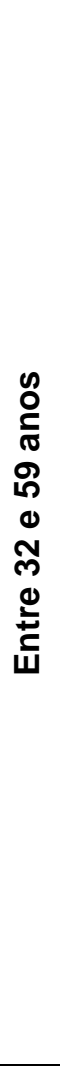 } & Sibilos ou chiado & 0,02 & $-0,01$ & 0,18 & 0,05 & 0,06 & $-0,06$ & $-0,08$ \\
\hline & $\begin{array}{l}\text { Estava associado a falta } \\
\text { de ar }\end{array}$ & 0,28 & 0,32 & 0,47 & 0,14 & 0,40 & 0,40 & 0,02 \\
\hline & $\begin{array}{l}\text { Presentes quando não } \\
\text { estava resfriado }\end{array}$ & 0,14 & $-0,05$ & 0,08 & 0,12 & 0,16 & $-0,10$ & 0,09 \\
\hline & $\begin{array}{l}\text { Acordou com a aperto ou } \\
\text { opressão }\end{array}$ & $-0,16$ & $-0,14$ & $-0,01$ & $-0,09$ & $-0,07$ & $-0,07$ & $-0,09$ \\
\hline & $\begin{array}{l}\text { Acordou com crise de falta } \\
\text { de ar }\end{array}$ & $-0,06$ & $-0,01$ & $-0,06$ & $-0,11$ & $-0,07$ & 0,02 & $-0,04$ \\
\hline & Acordou crise de tosse & $-0,11$ & $-0,04$ & 0,08 & $-0,09$ & $-0,03$ & $-0,04$ & $-0,12$ \\
\hline & Teve crise de asma & $-0,20$ & $-0,21$ & 0,09 & $-0,15$ & $-0,12$ & $-0,13$ & $-0,23$ \\
\hline & $\begin{array}{l}\text { Usando algum } \\
\text { medicamento para asma }\end{array}$ & $-0,20$ & $-0,21$ & 0,09 & $-0,15$ & $-0,12$ & $-0,13$ & $-0,23$ \\
\hline & $\begin{array}{l}\text { Alergia no nariz ou rinite } \\
\text { alérgica }\end{array}$ & $-0,11$ & $-0,11$ & 0,01 & 0,10 & $-0,12$ & $-0,20$ & $-0,17$ \\
\hline & $\begin{array}{l}\text { Tosse por pelo menos } 3 \\
\text { meses }\end{array}$ & $-0,19$ & $-0,11$ & 0,15 & $-0,12$ & $-0,08$ & $-0,14$ & $-0,23$ \\
\hline & $\begin{array}{l}\text { Catarro por pelo menos } 3 \\
\text { meses }\end{array}$ & 0,20 & 0,24 & 0,12 & 0,18 & 0,22 & 0,08 & 0,15 \\
\hline \multirow{7}{*}{ 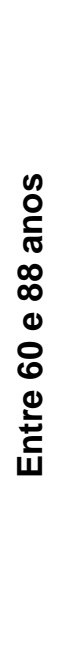 } & Sibilos ou chiado & $-0,08$ & $-0,07$ & 0,18 & $-0,02$ & $-0,03$ & $-0,09$ & $-0,17$ \\
\hline & $\begin{array}{l}\text { Estava associado a falta } \\
\text { de ar }\end{array}$ & 0,19 & 0,12 & 0,37 & 0,01 & 0,20 & 0,21 & $-0,18$ \\
\hline & $\begin{array}{l}\text { Presentes quando não } \\
\text { estava resfriado }\end{array}$ & $-0,19$ & $-0,20$ & $-0,34$ & $-0,47$ & $-0,27$ & $-0,05$ & 0,08 \\
\hline & $\begin{array}{l}\text { Acordou com a aperto ou } \\
\text { opressão }\end{array}$ & $-0,03$ & $-0,09$ & $-0,12$ & 0,04 & $-0,13$ & $-0,09$ & $-0,04$ \\
\hline & $\begin{array}{l}\text { Acordou com crise de falta } \\
\text { de ar }\end{array}$ & $-0,15$ & $-0,10$ & 0,05 & $-0,17$ & $-0,12$ & $-0,11$ & $-0,17$ \\
\hline & Acordou crise de tosse & $-0,07$ & $-0,06$ & 0,13 & $-0,01$ & 0,01 & $-0,10$ & $-0,10$ \\
\hline & Teve crise de asma & $-0,02$ & 0,14 & 0,03 & $0,38^{* *}$ & 0,12 & 0,16 & 0,11 \\
\hline
\end{tabular}




\title{
RECIMA21 - REVISTA CIENTÍFICA MULTIDISCIPLINAR
}

\author{
ASSOCIAC̄̃̃O ENTRE SINTOMAS RESPIRATÓRIOS, DADOS SOCIODEMOGRÁFICOS E \\ ANTROPOMETRIA EM AGRICULTORES FAMILIARES \\ lanka Quirino Soares dos Santos, Marta Ligia Vieira Melo, Kamilla Zenóbya \\ Ferreira Nóbrega de Souza, Elisangela Vilar de Assis, Ubiraídys de Andrade Isidório
}

Usando algum

medicamento para asma

0,20

0,16

$0,34^{\star}$

0,22

0,19

0,10

Alergia no nariz ou rinite alérgica

0,25

$0,30^{*}$

$0,30^{*}$

0,21

$0,32^{*}$

$-0,19$

$-0,11$

Tosse por pelo menos 3

meses

0,3

$0,25 \quad 0,35^{*}$

$0,25 \quad 0,30^{*}$

$\mathbf{0 , 2 4} \quad 0,06$

Catarro por pelo menos 3 meses

$\begin{array}{lllllll}0,01 & 0,02 & -0,02 & -0,07 & 0,01 & 0,04 & 0,03\end{array}$

Nota: Para os sintomas respiratório $0=$ não; $1=\operatorname{sim}$

A tabela 4 mostra que maior renda familiar bruta está relacionada a ausência de sintomas respiratórios (falta de ar presente quando não estava resfriado) para as pessoas com mais idade. Entre as pessoas mais jovens, verificou-se que maior escolaridade está relacionada a acordar com crise de tosse. Por fim, mais tempo de trabalho esteve relacionado a sibilos e tosse por pelo menos 03 meses.

Tabela 4 - Correlação de dados demográficos com sintomas respiratórios em função do sexo

\begin{tabular}{|c|c|c|c|c|c|c|c|}
\hline $\begin{array}{l}\stackrel{0}{0} \\
\text { g] }\end{array}$ & & 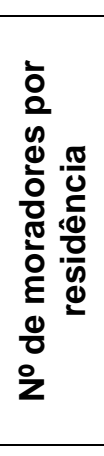 & 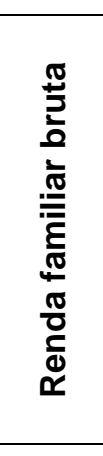 & $\begin{array}{l}\frac{0}{0} \\
\frac{\pi}{0} \\
\underline{0}\end{array}$ & 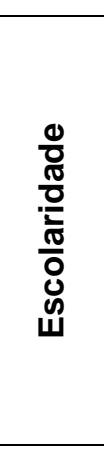 & 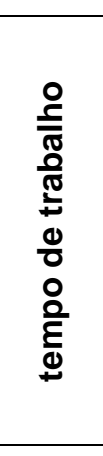 & 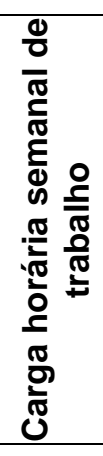 \\
\hline \multirow{8}{*}{ 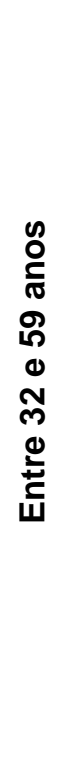 } & Sibilos ou chiado & $-0,08$ & 0,07 & 0,15 & 0,10 & $0,29 *$ & 0,10 \\
\hline & $\begin{array}{l}\text { Estava associado a falta de } \\
\text { ar }\end{array}$ & 0,32 & $-0,09$ & $-0,38$ & 0,09 & $-0,20$ & 0,14 \\
\hline & $\begin{array}{l}\text { Presentes quando não } \\
\text { estava resfriado }\end{array}$ & $-0,16$ & $-0,37$ & $-0,12$ & 0,09 & 0,13 & 0,37 \\
\hline & $\begin{array}{l}\text { Acordou com a aperto ou } \\
\text { opressão }\end{array}$ & 0,04 & $-0,21$ & $-0,14$ & $-0,16$ & 0,06 & 0,04 \\
\hline & $\begin{array}{l}\text { Acordou com crise de falta } \\
\text { de ar }\end{array}$ & 0,11 & 0,04 & $-0,19$ & 0,03 & $-0,01$ & $-0,06$ \\
\hline & Acordou crise de tosse & 0,02 & $-0,15$ & $-0,08$ & $0,40^{* *}$ & $-0,04$ & 0,06 \\
\hline & Teve crise de asma & $-0,02$ & $-0,11$ & $-0,01$ & $-0,03$ & 0,05 & 0,24 \\
\hline & $\begin{array}{l}\text { Usando algum medicamento } \\
\text { para asma }\end{array}$ & $-0,02$ & $-0,11$ & $-0,01$ & $-0,03$ & 0,05 & 0,24 \\
\hline
\end{tabular}




\begin{tabular}{|c|c|c|c|c|c|c|c|}
\hline & $\begin{array}{l}\text { Alergia no nariz ou rinite } \\
\text { alérgica }\end{array}$ & $-0,14$ & 0,07 & $-0,07$ & 0,01 & 0,19 & $-0,05$ \\
\hline & $\begin{array}{l}\text { Tosse por pelo menos } 3 \\
\text { meses }\end{array}$ & $-0,19$ & $-0,02$ & 0,19 & 0,03 & $0,27^{\star}$ & 0,01 \\
\hline & $\begin{array}{l}\text { Catarro por pelo menos } 3 \\
\text { meses }\end{array}$ & $-0,23$ & 0,03 & 0,13 & 0,04 & $-0,23$ & 0,04 \\
\hline & Sibilos ou chiado & 0,05 & $-0,05$ & $-0,16$ & $-0,07$ & ** & 0,20 \\
\hline & $\begin{array}{l}\text { Estava associado a falta de } \\
\text { ar }\end{array}$ & 0,13 & $-0,49^{*}$ & $-0,44$ & 0,27 & $\star *$ & $-0,04$ \\
\hline & $\begin{array}{l}\text { Presentes quando não } \\
\text { estava resfriado }\end{array}$ & $-0,25$ & $-0,49^{*}$ & $-0,33$ & $-0,11$ & $\star * *$ & $-0,04$ \\
\hline & $\begin{array}{l}\text { Acordou com a aperto ou } \\
\text { opressão }\end{array}$ & 0,18 & $-0,07$ & 0,07 & 0,01 & $\star *$ & $-0,13$ \\
\hline 유 & $\begin{array}{l}\text { Acordou com crise de falta } \\
\text { de ar }\end{array}$ & $-0,08$ & $-0,33^{*}$ & $-0,18$ & 0,01 & ** & $-0,13$ \\
\hline 0 & Acordou crise de tosse & 0,19 & 0,20 & 0,12 & $-0,23$ & $\star \star *$ & 0,24 \\
\hline 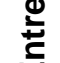 & Teve crise de asma & $-0,09$ & 0,04 & $-0,16$ & 0,04 & ** & $-0,10$ \\
\hline & $\begin{array}{l}\text { Usando algum medicamento } \\
\text { para asma }\end{array}$ & 0,26 & 0,17 & $-0,01$ & 0,19 & ** & $-0,14$ \\
\hline & $\begin{array}{l}\text { Alergia no nariz ou rinite } \\
\text { alérgica }\end{array}$ & 0,09 & 0,07 & $-0,24$ & $-0,26$ & ** & 0,10 \\
\hline & $\begin{array}{l}\text { Tosse por pelo menos } 3 \\
\text { meses }\end{array}$ & $-0,08$ & 0,01 & $-0,14$ & 0,09 & ** & 0,06 \\
\hline & $\begin{array}{l}\text { Catarro por pelo menos } 3 \\
\text { meses }\end{array}$ & $-0,16$ & $-0,01$ & 0,16 & 0,08 & ** & 0,13 \\
\hline
\end{tabular}

Nota: Para os sintomas respiratório 0 = não; 1 = sim; ** todos os idosos possuem mais de 20 anos de trabalhos, por isso não foi possível correlacionar

\section{DISCUSSÃO}

Os resultados obtidos pela pesquisa caracterizam os participantes a partir de idade entre 32 a 88 anos, alguns são alfabetizados, os mais jovens recebem menos de um salário mínimo e os idosos recebem dois. Algumas pessoas trabalham há mais de 20 anos e com carga horária de trabalho semanal de 60hrs.

Foram mensuradas suas medidas antropométricas e os dados demográficos, correlacionando-os com os sintomas respiratórios. Nas pessoas mais jovens não foi encontrada correlação e nas mais velhas foi encontrada correlação em alguns sintomas de alergias respiratórias. 


\title{
RECIMA21 - REVISTA CIENTÍFICA MULTIDISCIPLINAR
}

\author{
ASSOCIAÇÃO ENTRE SINTOMAS RESPIRATÓRIOS, DADOS SOCIODEMOGRÁFICOS E \\ ANTROPOMETRIA EM AGRICULTORES FAMILIARES \\ lanka Quirino Soares dos Santos, Marta Ligia Vieira Melo, Kamilla Zenóbya \\ Ferreira Nóbrega de Souza, Elisangela Vilar de Assis, Ubiraídys de Andrade Isidório
}

Peres et al. (2019) alertam que é muito importante um ambiente seguro e adequado para os trabalhadores, e que deve ser realizada uma avaliação em relação as exposições ocupacionais. Petarli et al., (2019) relatam que grande parte dos agricultores trabalham na agricultura há mais de 20 anos, com jornada laboral de 40 horas semanais, ficando expostos por vários tipos de fatores de riscos à saúde, como poeira, fumaça, e agrotóxicos, e a maioria se recusa a utilizar os Equipamentos de Proteção Individual (EPIs) durante o trabalho, justificando o incômodo para não utilização.

Santana et al. (2016) apontam que uma parte dos agricultores não concluem o ensino médio, e a outra são de pessoas analfabetas. O baixo nível de escolaridade dificulta o entendimento das informações sobre o uso dos EPIs, e até mesmo a aplicação dos agrotóxicos, os quais são aplicados sem a adequada leitura dos rótulos. Petarli et al. (2019) dizem que metade dos agricultores não leem o rótulo dos agrotóxicos, não observam o tempo para a reaplicação e muito menos cumprem o tempo adequado para aproximação com a lavoura, e infelizmente isso faz muito mal à saúde deles.

Bião et al. (2017) ressaltam que o baixo nível de escolaridade dos agricultores brasileiros é uma realidade triste do país, pois suas condições trabalhistas são indigentes, porque pela falta de opção de trabalho acabam trabalhando em ambientes que os tornam vulneráveis, com sérios efeitos a saúde. Petarli et al (2019) afirmam que até para aquisição dos agrotóxicos, os agricultores contam com a indicação de vizinhos, amigos e familiares, comprando de forma livre em lojas de produtos agropecuários. Santana et al. (2016) afirmam que essa população é muito despreparada para utilização dessas substâncias, pois correm o risco de sofrerem intoxicações.

Dias; Bronhara; Calamita (2014) dizem que a saúde pública brasileira passa por um grande desafio, que é o envelhecimento da população, em que as pessoas são consideradas idosas com idades acima de 60 anos, e a maioria têm o nível socioeconômico e educacional baixo. Nos últimos anos, foi visto um grande crescimento do número de doenças respiratórias em idosos, pois eles possuem uma grande sensibilidade, causando assim o aumento das alergias.

Cruz et al. (2018) relatam que na terceira idade é normal acontecer algumas alterações estruturais que podem dificultar a reexpansão pulmonar, como o achatamento do diafragma, causando a diminuição da capacidade de gerar pressão intratorácica negativa, e é comum uma redução significativa da força dos músculos respiratórios, não só devido às alterações anatômicas, mas também a défices nutricionais frequentes nesta faixa etária.

Dias; Bronhara; Calamita (2014) esclarecem que não foram encontradas diferenças significativas na prevalência de asma e rinite entre pessoas idosas e não idosas e que a sensibilidade a ácaros que causam as doenças alérgicas atinge tanto os idosos quanto os jovens. Cruz et al. (2018) revelam que a idade não tem influência significativa na resistência das vias respiratórias, mas na terceira idade é importante uma atenção maior com os riscos de rinite e tosse, por afetarem negativamente o trato respiratório, deixando as narinas ressecadas e desenvolvendo 


\title{
RECIMA21 - REVISTA CIENTÍFICA MULTIDISCIPLINAR
}

\author{
ASSOCIAÇÃO ENTRE SINTOMAS RESPIRATÓRIOS, DADOS SOCIODEMOGRÁFICOS E \\ ANTROPOMETRIA EM AGRICULTORES FAMILIARES \\ lanka Quirino Soares dos Santos, Marta Ligia Vieira Melo, Kamilla Zenóbya \\ Ferreira Nóbrega de Souza, Elisangela Vilar de Assis, Ubiraídys de Andrade Isidório
}

muco nas vias aéreas, e é comum eles já fazerem uso de fármacos, prejudicando ainda mais sua qualidade de vida.

Rasslan et al, (2009) mostram que a obesidade afeta o tórax e o diafragma, determinando alterações na função respiratória, devido ao aumento do esforço respiratório e comprometimento no sistema da troca dos gases. Silveira; Vieira; Danesio (2018) relatam que pessoas idosas e não idosas que são obesas devem dar importância para saúde, pois pode comprometer a função pulmonar e o padrão ventilatório, independente se a pessoa fuma ou não, ou seja, se for fumante os riscos são maiores. Tudo isso pode ser resultado tanto de efeitos mecânicos quanto de efeitos metabólicos do tecido adiposo.

Rasslan et al., (2009) afirmam que as alterações respiratórias ocorrem de acordo com o grau de obesidade, ou seja, quanto mais obeso o indivíduo for mais dificuldades respiratórias ele terá. Soares et al. (2011) ressaltam que a obesidade influencia muito na capacidade física e respiratória, independentemente de qualquer idade, e afirma que, além de fazer com que ocorra compressão do tórax, do diafragma e do pulmão, o que ocasiona a redução dos volumes pulmonares e acarretar alterações musculares, também causa a redução do bem-estar psíquico, como a dificuldade de interação social até mesmo no ambiente de trabalho.

Sonehara et al. (2011) mostram que o sedentarismo é um estilo de vida muito comum causado pela obesidade, que é responsável pelos altos índices de insucessos e pelo aparecimento de muitas doenças, o que pode levar ao surgimento de transtornos psicossociais, prejudicando a qualidade de vida e dificultando sua produtividade no trabalho e a relação com outras pessoas.

Bião et al. (2017) afirmam que as patologias respiratórias acontecem em todas as idades, tanto em pessoas idosas e não idosas, e podem ser causadas pelos diversos tipos de atividades que o trabalho na agricultura oferece. Alves et al. (2019) apontam que muitos trabalhadores exercem durante anos as mesmas funções, e ainda esclarecem que a inalação de fumaça, poeira e agrotóxicos, decorrente da não utilização de EPIs, causam pneumocominoses como, DPOC e asma ocupacional, com isso os agricultores podem apresentar sintomas significativos como: tosse produtiva constante, fadiga, dispneia e dores no corpo. E nas formas avançadas eles podem apresentar dispneia aos mínimos esforços, tosse com catarro, diminuição do murmúrio vesicular, sibilos e roncos.

\section{CONIDERAÇÕES FINAIS}

A maioria dos agricultores foi constituída por indivíduos não-alfabetizados, que tinham mais de 20 anos de trabalho na agricultura, com carga horária entre 40 e 50h semanais. Com relação aos dados antropométricos, os valores entre o grupo de agricultores jovens e idosos foram próximos. 


\title{
RECIMA21 - REVISTA CIENTÍFICA MULTIDISCIPLINAR
}

\author{
ASSOCIAÇ̃̃O ENTRE SINTOMAS RESPIRATÓRIOS, DADOS SOCIODEMOGRÁFICOS E \\ ANTROPOMETRIA EM AGRICULTORES FAMILIARES \\ lanka Quirino Soares dos Santos, Marta Ligia Vieira Melo, Kamilla Zenóbya \\ Ferreira Nóbrega de Souza, Elisangela Vilar de Assis, Ubiraídys de Andrade Isidório
}

No que se refere aos sintomas respiratórios observou-se que no grupo dos agricultores houve associação entre os sintomas respiratórios (teve crise de asma, uso de algum medicamento para asma, alergia no nariz ou rinite alérgica e tosse por pelo menos três meses) e variáveis antropométricas.

Tempo de trabalho e escolaridade e presença de sintomas respiratórios foram associados no grupo dos agricultores mais jovens, enquanto no grupo dos idosos essa associação foi observada com a renda familiar bruta.

Portanto, constata-se a necessidade de assistência para a saúde desses trabalhadores, com estratégias preventivas que objetivem a conscientização dessa população, fazendo com que os números de patologias respiratórias decorrentes do ambiente de trabalho sejam reduzidas.

\section{REFERÊNCIAS}

ALVES, B. P. V. et. al. Pneumoconiose em trabalhadores de carvoaria: um relato de caso. Revista Eletrônica Acervo Saúde, v. 19, Supl. 9, p.1-5, 2019.

BIÃO, M. A. S. et al. Prevalência de sinais e sintomas respiratórios associados á asma em trabalhadores da indústria canavieira no Recôncavo Baiano. Revista Diálogo e Ciências, v. 39, p. 116, 2017.

BORGES, A. et al. Agricultura familiar e a conservação da saúde humana e ambiental. Revista Brasileira de Enfermagem, v. 69, n. 2, p. 304-12, 2016.

BRASIL. Casa Civil. Secretaria Especial de Agricultura Familiar e do Desenvolvimento Agrário (SEAD). Extrato DAP. 2019. Disponível em: http://smap14.mda.gov.br/extratodap/PesquisarDAP. Acesso em: 14 maio 2019.

BRASIL. Lei n. 11.326, de 24 de julho de 2006. Estabelece as diretrizes para a formulação da Política Nacional da Agricultura Familiar e Empreendimentos Familiares Rurais. Disponível em: http://www.planalto.gov.br/ccivil 03/ Ato2004-2006/2006/Lei/L11326.htm. Acesso em: 12 maio 2020.

BRASIL. Ministério da Saúde. Conselho Nacional de Saúde. Comissão Nacional de Ética em Pesquisa. Resolução n. 466/12, de 12 de dezembro de 2012. Brasília: Ministério da Saúde, 2012.

BURALLI, R. Avaliação da condição respiratória em população rural exposta a agrotóxicos no município de São José de Ubá, Estado do Rio de Janeiro. Tese (Doutorado) - São Paulo, Universidade de São Paulo, 2016.

CRUZ, C. et al. Doença alérgica respiratória no idoso. Revista Portuguesa de Imunoalergologia, v. 26, n. 3, p. 189-205, 2018.

DIAS, L.; BRONHARA, A.; CALAMITA, Z. Estudo comparativo sobre a prevalência de alergias entre idosos e não idosos. Jornal Brasileiro de Alergia e Imunologia, v. 2, n. 2, p. 75-85, 2014.

FARIAS, K. Caracterização do hemograma e perfil bioquímico sérico de agricultores fumageiros expostos a agrotóxicos na área rural de Arapiraca - AL. Dissertação (Programa de Pós-Graduação em Ciências da Saúde) - Instituto de Ciências Biológicas e da Saúde, Maceió, Universidade Federal de Alagoas, 2012. 


\title{
RECIMA21 - REVISTA CIENTÍFICA MULTIDISCIPLINAR
}

\author{
ASSOCIAÇ̃̃O ENTRE SINTOMAS RESPIRATÓRIOS, DADOS SOCIODEMOGRÁFICOS E \\ ANTROPOMETRIA EM AGRICULTORES FAMILIARES \\ lanka Quirino Soares dos Santos, Marta Ligia Vieira Melo, Kamilla Zenóbya \\ Ferreira Nóbrega de Souza, Elisangela Vilar de Assis, Ubiraídys de Andrade Isidório
}

FERRAZ, L. et al. As demandas do homem rural: informações para a assistência nos serviços de saúde da atenção básica. Revista Mineira de Enfermagem, v. 17, n. 2, p. 349-355, 2013.

FONTOURA, G.; ZANAGA, A. Proteção da saúde de trabalhadores rurais: a necessidade de padronização das metodologias de quantificação da exposição dérmica a agrotóxicos. Cadernos de Saúde Pública, v. 30, n. 5, p. 952-960, 2014.

FREITAS, F. Avaliação da prevalência de sintomas respiratórios em trabalhadores rurais expostos ao uso de agrotóxicos no Vale de Cuncas, Barro-CE. Dissertação (Mestrado em Saúde Coletiva) - Santos, Universidade Católica de Santos, 2017.

GREGOLIS, T.; PINTO, W.; PERES, F. Percepção de riscos do uso de agrotóxicos por trabalhadores da agricultura familiar do município de Rio Branco, AC. Revista Brasileira de Saúde Ocupacional, v. 37, n. 125 , p. $99-113,2012$.

GUIMARÃES, D. G.; OLIVEIRA, S. P. Análise de utilização e percepção de risco no uso de agrotóxicos pelos agricultores familiares do município de malhada de pedras BA. Enciclopédia Biosfera, Goiânia, v. 11, p. 11-15, 2015.

HOEPERS, A. T. C. Prevalência de multimorbidade na população de Florianópolis com idade igual ou superior a 40 anos: clusters e networking das morbidades. Tese (Doutorado) - Centro de Ciências da Saúde, Florianópolis, Universidade Federal de Santa Catarina, 2015.

LISBOA, D.; AZEVEDO, E. Cultivo do tabaco no sul do Brasil: doença da folha verde e outros agravos à saúde no Sul do Brasil, Ciência e Saúde coletiva, v.19, n.12, p.4797-4808, 2014.

MOREIRA, J. P. L. et al. A saúde dos trabalhadores da atividade rural no Brasil. Caderno de Saúde Pública, v. 31, n. 8, p. 1698-1708, 2015.

PETARLI, G. B. et al. Exposição ocupacional a agrotóxicos, riscos e práticas de segurança na agricultura familiar em município do estado do Espírito Santo, Brasil. Revista Brasileira de Saúde Ocupacional, v. 44, n. 15, p. 1-13, 2019.

RASSLAN, Z. et al. Função pulmonar e obesidade. Revista da Sociedade Brasileira Clínicas Médicas, v. 7, p. 36-39, 2009.

RONDON, E.; GONÇALVES, R.; BOTELHO, C. Sintomas respiratórios como indicadores de estado de saúde em trabalhadores de indústrias de cerâmicas. Jornal Brasileiro de Pneumologia, v. 37, n. 1, p. 36-45, 2011.

SANTANA, C. M. et al. Exposição ocupacional de trabalhadores rurais a agrotóxicos. Caderno de Saúde Coletiva, v. 24, n. 3, p. 301-307, 2016.

SILVEIRA, E.; VIEIRA, L.; DANESIO, J. Elevada prevalência de obesidade abdominal em idosos e associação com diabetes, hipertensão e doenças respiratórias. Ciência \& Saúde Coletiva, v. 23, n. 3, p. 903-912, 2018.

SOARES, K. K. D. et al. Avaliação do desempenho físico e funcional respiratório em obesos. Fisioterapia em Movimento, v. 24, n. 4, p. 697-704, 2011.

SONEHARA, E. et al. Efeitos de um programa de reabilitação pulmonar sobre mecânica respiratória e qualidade de vida de mulheres obesas. Fisioterapia em Movimento, v. 24, n. 1, p. 13-21, 2011. 